\title{
Our experience with laparoscopic total gastrectomy for gastric cancer: A case series
}

\author{
Manash Ranjan Sahoo ${ }^{1 *}$, Satyajit Samal ${ }^{2}$, Jyotirmay Nayak ${ }^{2}$ \\ ${ }^{1}$ All India Institute of Medical Sciences (AIIMS), Bhubaneswar, Odisha, India. \\ ${ }^{2}$ Department of Surgery, S.C.B. Medical College \& Hospital, Cuttack, Odisha, India
}

\section{To Cite}

Sahoo MR, Samal S, Nayak J. Our experience with laparoscopic total gastrectomy for gastric cancer: A case series. J Gastric Surg 2020; 2(3): 60-65

\section{Publication history}

Received: July 31, 2020

Accepted: August 6, 2020

Article in press: August 20, 2020

Published online: August 28, 2020

\section{*Correspondence to}

Dr. Manash Ranjan Sahoo

Professor and Head,

Department of Surgery

All India Institute of Medical Sciences (AIIMS)

Bhubaneswar, Odisha, India.

manash67@gmail.com

\begin{abstract}
Background:

Though laparoscopic distal gastrectomy has become a standard procedure for treatment of gastric cancer, laparoscopic total gastrectomy has not been widely accepted as it requires more dexterity and lack of evidence about its feasibility and safety.

Methods:

Here retrospectively we review a series of 12 cases of gastric cancer undergone laparoscopic total gastrectomy with D1 or D2 lymphadenectomy over a period of 10 years at a tertiary care hospital. The patient demographic characteristics were reviewed and the outcomes after surgery was analyzed in terms of extent of lymphadenectomy, mean operative time, mean intraoperative blood loss median number of lymph nodes harvested, median time for postoperative ambulation, median time for postoperative oral feeding, median time of postoperative hospital stay, postoperative complications and mortality.
\end{abstract}

\section{Results:}

All patients had total gastrectomy entirely through laparoscopic method. Mean operative time was 282 minutes, mean intraoperative blood loss was $120 \mathrm{ml}$, median time for ambulation and oral feeding was 3 days and 6 days respectively. Median time of hospital stay was 16 days and 2 patients had complications as pancreatic fistula and port site abscess. No mortality was observed.

\section{Conclusion:}

With zero mortality and accepted rate of complications, laparoscopic total gastrectomy appears to be technically feasible and safe for management of gastric cancer. But more studies have to be conducted with comparison to other standard gastrectomies and long term follow up to be done to establish its standardized application Keywords:

gastric cancer, laparoscopy, total gastrectomy. 


\section{Background:}

Gastric cancer is still a dreaded malignancy, being the fourth most common cancer and the third leading cause of cancer-related death worldwide [1]. Controversy persists regarding the laparoscopic approach for various gastric oncological procedures, although support and evidence for the minimally invasive approach is growing [2,3]. In 1994, Kitano performed the first laparoscopic assisted distal gastrectomy with a modified D1 lymph node dissection for the treatment esophago-gastric cancer with high risk of lymph node metastasis. Laparoscopyassisted distal gastrectomy has become the recognized procedure for early gastric cancer [4-6]. Its benefits over conventional open gastrectomy, such as improved cosmetics and reduced postoperative pain, have been well documented. But unlike this, laparoscopic total gastrectomy remains a challenging procedure and the technique has not yet been standardized [7]. This is partly related to skepticism regarding oncologic results for this advanced surgical procedure with a significant learning curve. Laparoscopic gastrectomy is a well-established technique for treating gastric cancer in Eastern countries. According to national surveys, LG accounted for approximately $25 \%$ of all gastric surgeries performed for cancer in Japan and South Korea as early as 2009 [8]. In 1996, Azagra et al reported the first laparoscopic total gastrectomy for cancer There are few studies on the outcomes of laparoscopic total gastrectomy with D1 or D2 lymphadenectomy for gastric cancer because of technical difficulty in completing the procedures and concerns about long-term oncologic outcomes. The application of laparoscopic total gastrectomy with D2 lymphadenectomy for gastric cancer raises 2 problems: whether and how to perform a concomitant splenectomy to completely retrieve station 10 lymph nodes and how to accomplish an intracorporeal anastomosis. The former is also a matter of debate for open surgery, and the latter is the key to success for a totally laparoscopic approach. Herein we report our experience with the feasibility, safety and postoperative outcomes of 12 cases of gastric cancer undergone laparoscopic total gastrectomy with lymphadenectomy by a single surgeon and his team.

\section{Methods:}

Total 12 patients of gastric cancer had undergone laparoscopic total gastrectomy with D1 and D2 lymphadenectomy at S.C.B. Medical College and Hospital, Cuttack \& AIIMS Bhubaneswar, Odisha from March 2010 to July 2019. Retrospectively the details of their treatment and hospital stay was retrieved from the computerized patient database. The data was collected regarding age, sex, height, weight, body mass index (BMI), clinical and pathological stage, extent of gastrectomy and lymph node dissection, operative time, intraoperative blood loss, postoperative hospital stay, oral intake rate, intraoperative and postoperative complications, mortality and re-admission rate. The patient and surgical characteristics are given in Table 1. All patients were found to be histologically diagnosed as adenocarcinoma of stomach of various stages and had undergone laparoscopic total gastrectomy and Roux-
en-Y reconstruction with a stapled esophagojejunostomy.

\begin{tabular}{|l|l|}
\hline Median age (years) & $58.6 \pm 8.2(42-69)$ \\
\hline Sex & 11 \\
\hline Male & 1 \\
\hline Female & $19.2(17.4-31.7)$ \\
\hline Mean BMI (Body Mass Index) & \multicolumn{2}{|l|}{} \\
\hline American society of Anaesthesiologists class \\
\hline ASA 1 & 0 \\
\hline ASA 2 & 8 \\
\hline ASA 3 & 4 \\
\hline Location of gastric cancer & \multicolumn{2}{|l|}{} \\
\hline Whole & 4 \\
\hline Upper & 6 \\
\hline Middle & 2 \\
\hline
\end{tabular}

Table 1: Patient characteristics.

Operative intervention was done as D1 to D1+ resection for early gastric cancer and D2 resection was performed for advanced gastric cancer (Figure 1) according to the criteria of gastric cancer treatment guidelines of JGCA [9]. The standard operative procedure followed was as below.

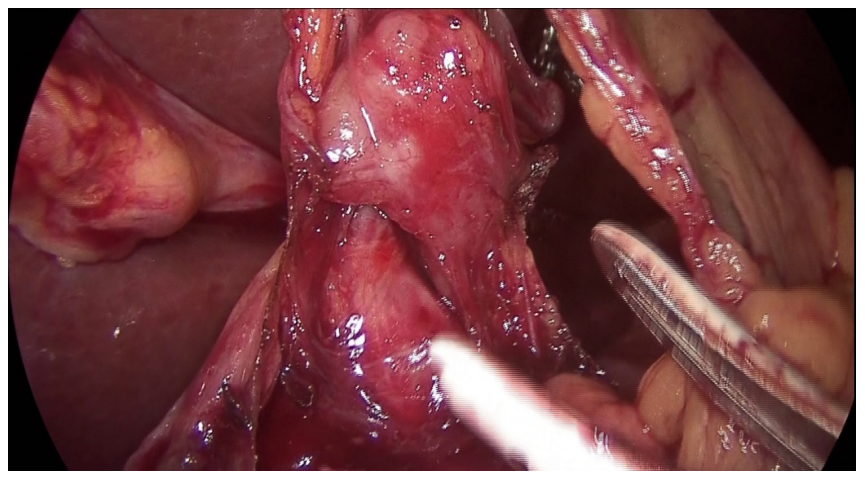

Figure 1: Lymphadenectomy.

After the establishment of a pneumoperitoneum at 10 to $12 \mathrm{~mm} \mathrm{Hg}$, a laparoscope was introduced through the infraumbilical port. The other 4 trocars, inserted under laparoscopic guidance, consisted of two 5-mm bilateral subcostal ports and two 12-mm bilateral low abdominal ports. At the beginning of each operation, the peritoneal cavity was carefully inspected and cytologic examination by peritoneal lavage was performed to detect macroscopic or microscopic peritoneal dissemination of tumor cells.

An avascular area of the gastrocolic ligament was divided toward the lower pole of the spleen by means of laparosonic coagulation shears and all lymph nodes along the gastroepiploic vessels (stations $4 \mathrm{~d}$ and $4 \mathrm{sb}$ ) were dissected, and then the division was extended distally toward the pylorus by means of laparosonic coagulation shears. The right gastroepiploic vessels were exposed and divided with a single clip at their origins, thus allowing dissection of the infrapyloric lymph nodes (station 6) from the pancreatic head.

The peritoneum along the inferior pancreatic margin was incised with an electrocautery. The body of the gland was lifted from its bed and further mobilized as far as necessary, and the splenic vein and artery were 
isolated. The caudal gland of the pancreas was retracted gently downward by gauze traction so that the upper border of the pancreas was moved forward. The splenic artery was immediately identified through the lesser sac and was divided between clips distal to the origin of the dorsal pancreatic artery.

The lower esophagus was adequately mobilized (Figure 2, Figure 3) and transected (Figure 4) by means of a 45mm endoscopic stapling device.

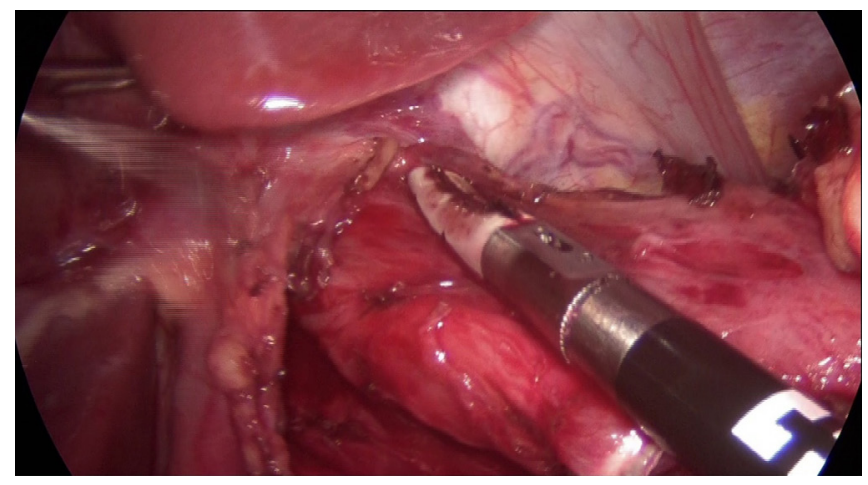

Figure 2: Hiatal dissection.

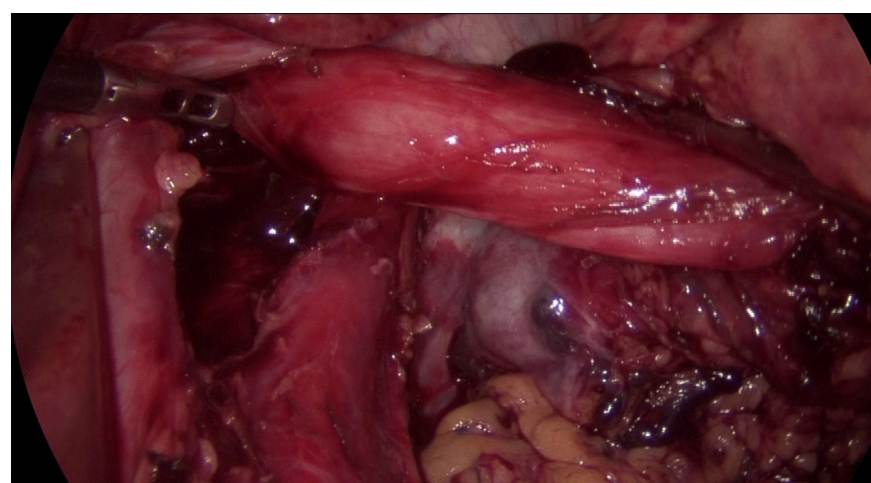

Figure 3: Mobilised oesophagus.

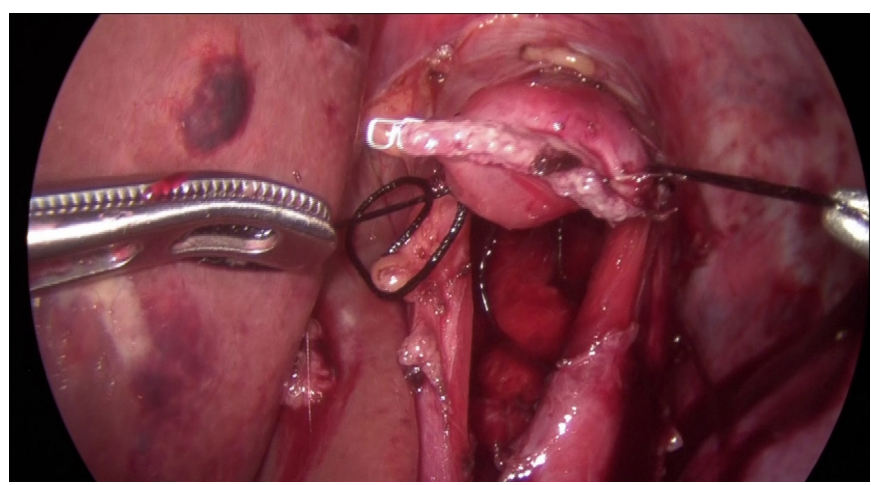

Figure 4: Oesophageal transection.

The stumps of the excised portion of the esophagus were submitted for frozen-section examination with hematoxylin-eosin staining during the operation in patients with intraoperative suspected tumor invasion of the esophagus. The whole stomach and the body and tail of the pancreas, together with the spleen, were turned over to the right, exposing their posterior surfaces. The fatty connective tissue including lymph nodes around the splenic artery (stations 11p and 11d) was completely removed and the caudal splenic artery was divided with clips. The splenic vein was preserved on the whole length of the pancreas and divided between clips just distal to the point of entry to the pancreas tail. The celiac and left gastric arteries were approached from the left and behind. The root of the left gastric artery was divided with double clips (Figure 5), followed by dissection of lymph nodes along the celiac artery (station 9) and the left gastric artery (station 7).

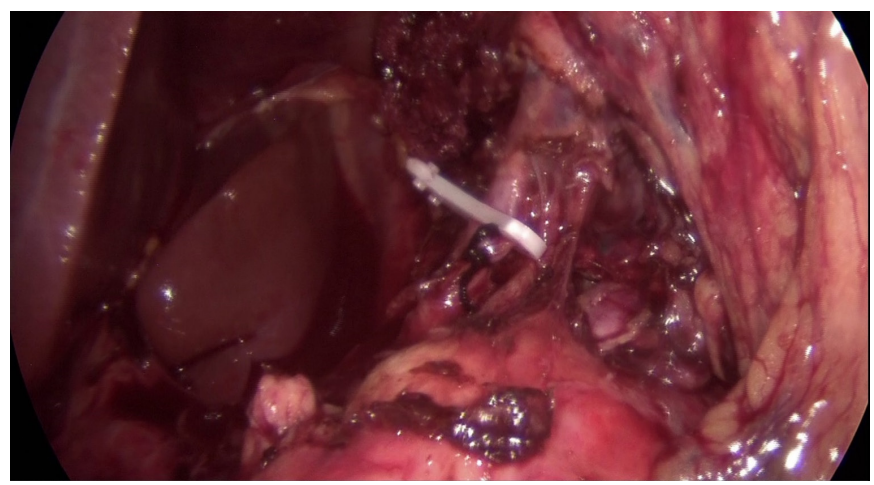

Figure 5: Managing left gastric vessels.

The whole of the stomach was turned down and the duodenum was transected at $1 \mathrm{~cm}$ distal to the pylorus with the endoscopic stapling device (Figure 6).

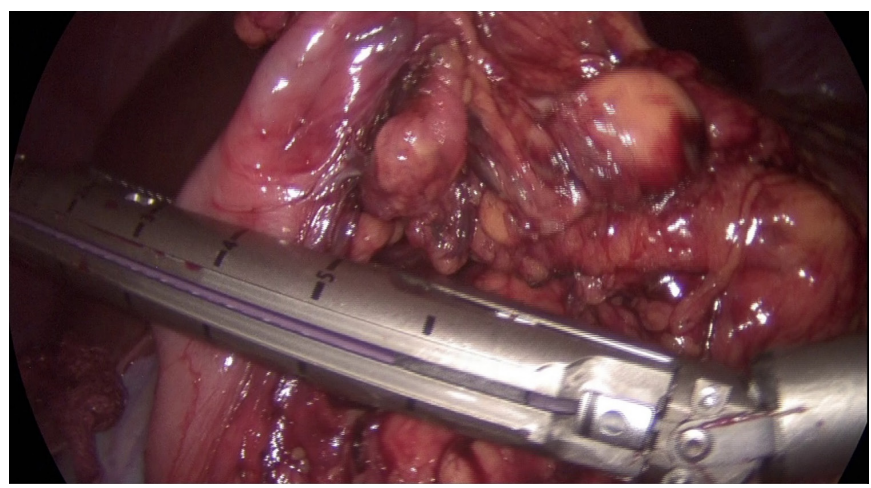

Figure 6: Duodenal division.

The hepatoduodenal ligament was dissected, and then the right gastric artery was exposed and divided at its origin, allowing dissection of the suprapyloric lymph nodes (station 5). The lymph nodes along the common hepatic (station 8a) and proper hepatic (station 12a) arteries were removed en bloc just anterior to the portal vein. Finally, the resected organs and lymph nodes were placed in a plastic bag and extracted through the umbilical incision extended vertically for $40 \mathrm{~mm}$. A proximal margin of at least $2 \mathrm{~cm}$ is recommended for T1 tumors, $3 \mathrm{~cm}$ is recommended for T2 or deeper tumors with an expansive growth pattern (Bormann I and II), and $5 \mathrm{~cm}$ is recommended for those with infiltrative growth pattern (Bormann III and IV).

After total gastrectomy, intracorporeal anastomosis was accomplished by the Roux-en-Y method with a functional side-to-side anastomotic technique. The ligament of Treitz was identified and the jejunum was divided at $20 \mathrm{~cm}$ distally. The distal limb of the jejunum was brought up in an antecolic fashion. An enterotomy was made by electrocautery in the antimesenteric side of the jejunum and the posterior side of the esophagus to allow passage of the 2 forks of the endoscopic stapling device. The stapling device was closed and fired to create the anastomosis (Figure 7). The connected stab incision was closed with 2 or 3 applications of the stapler (Figure 8). The side-to-side enteroenterostomy to the Roux limb 
was made at $50 \mathrm{~cm}$ below the esophagojejunostomy by means of the same stapling technique (Figure 9).

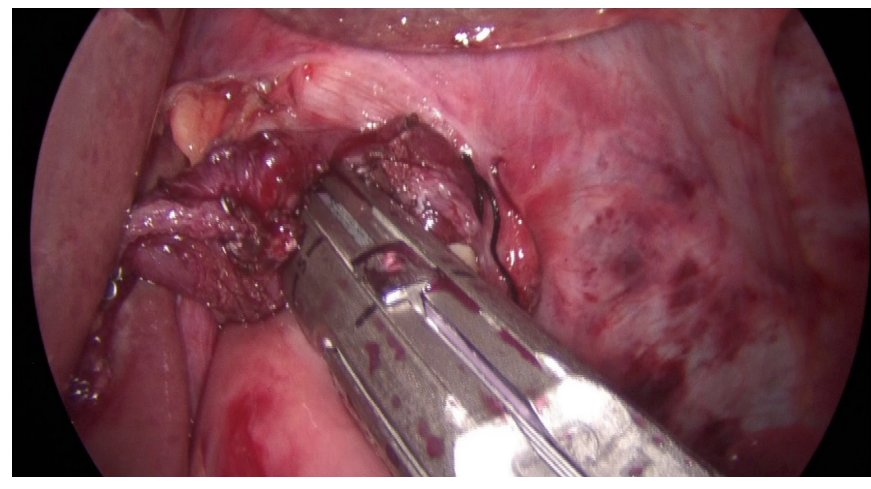

Figure 7: Oesophagojejunostomy.

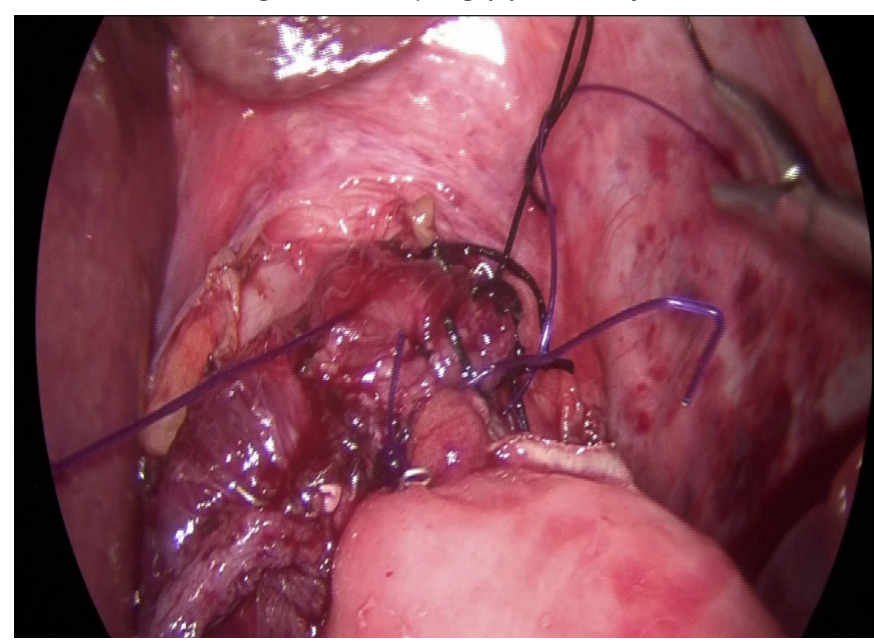

Figure 8: Oesophagojejunostomy stoma closure.

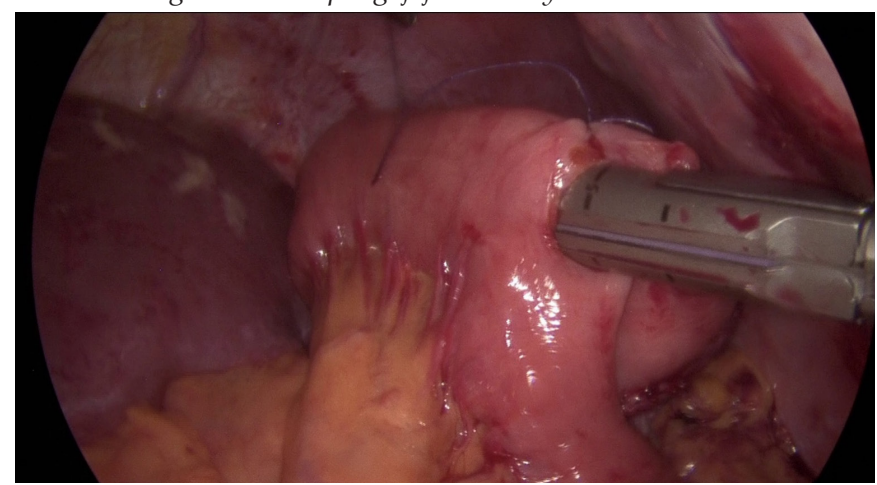

Figure 9: Enteroenterostomy.

Two soft tube drains were usually placed, one in the left subphrenic space and another near the esophagojejunostomy through separate bilateral ports, and left for 3 or 4 days.

After discharge from our hospital all the patients had undergone oncological evaluation at regional cancer center to receive postoperative adjuvant chemotherapy and/or radiotherapy.

\section{Results}

There were 11 males (91.7\%) and 1 female (8.3\%) with a median age of 58.6 years (range $42-69$ years). All of them had comorbidities with 8 patients $(75 \%)$ under ASA class II and 4 patients (25\%) under ASA class III. No patient had history of any previous laparoscopic surgery or laparotomy. The surgical characteristics were as given in Table 2 . In clinicopathological staging, 3 patients were under stage IA, 4 under stage IB, 2 under stage IIA, 1 under stage IIB and 2 patients under stage III (according to the International Union Against CancerTNM staging).

All the procedures were accomplished entirely through laparoscopic approach. The mean operative time was 282 minutes (range 230-465 minutes). The mean intraoperative blood loss was $120 \mathrm{ml}$ (range 50-650 ml) and blood transfusion was needed for 2 patients during surgery, with 1 unit for each. D1 lymphadenectomy was done in 3 patients, D1+ lymphadenectomy in 5 patients, D2 lymphadenectomy was done in 4 patients and 1 patient had undergone concurrent splenectomy. The median number of lymph nodes harvested was 26 (range 18-42) and positive lymph node metastasis was found in 9 patients. The median time period of postoperative ambulation was 3 days (range 2-5 days) and 6 days (range 5-8 days) for postoperative oral feeding. The median duration of postoperative hospital stay was 16 days (range 13- 83 days). 10 patients had uneventful postoperative hospital stay, 2 patients had complications leading to longer hospital stay. Out of 12 patients, 2 had developed complications. The patient with concurrent splenectomy developed pancreatic fistula which was managed conservatively with keeping the drain for longer time which was placed during laparoscopic total gastrectomy and CT guided percutaneous drainage later. Another patient developed port site abdominal wall abscess which was managed conservatively and local abscess site exploration. No patient had undergone abdominal re-explorative surgery. No other postoperative complication was observed. There was no mortality during in-hospital stay and 30 days after discharge till 2nd follow up visit of the patients. Table 2.

\begin{tabular}{|c|c|}
\hline \multicolumn{2}{|l|}{ Stage } \\
\hline IA & 3 patients \\
\hline IB & 4 patients \\
\hline II & 2 patients \\
\hline IIB & 1 patient \\
\hline III & 2 patients \\
\hline IV & 0 patients \\
\hline \multicolumn{2}{|l|}{ Extent of lymphadenectomy } \\
\hline D1 & 3 patients \\
\hline $\mathrm{D} 1+$ & 5 patients \\
\hline D2 & 4 patients \\
\hline Mean operative time (minutes) & $\begin{array}{l}282 \pm 37.5(230- \\
465)\end{array}$ \\
\hline Mean intraoperative blood loss (ml) & $120 \mathrm{ml}(50-650)$ \\
\hline Median number of lymph nodes harvested & $26(18-42)$ \\
\hline Positive lymph node metastasis & 9 patients \\
\hline Median time for postoperative ambulation & 3 days ( $2-5$ days) \\
\hline Median time for postoperative oral feeding & 6 days (5-8 days) \\
\hline Median time of postoperative hospital stay & $\begin{array}{l}16 \text { days (13-83 } \\
\text { days) }\end{array}$ \\
\hline \multicolumn{2}{|l|}{ Postoperative complication } \\
\hline Pancreatic fistula & 1 patient \\
\hline Port site abscess & 1 patient \\
\hline
\end{tabular}

Table 2: Surgical Characteristics. 


\section{Discussion:}

Recently, several studies on laparoscopic total gastrectomy for gastric cancer have shown not only favorable short-term outcomes but also acceptable longterm oncologic safety $[10,11]$. In contrast to laparoscopic partial gastric resections for early gastric cancer, studies on laparoscopic total gastrectomy for gastric cancer are lacking [12,13]. In this case series report we have successfully performed 12 laparoscopic total gastrectomies with a complete D1 or D2 lymph node dissection without conversion to open laparotomy. To the best of our knowledge, this is the first report on the outcomes of laparoscopic total gastrectomy on a series of gastric cancer patients in the state.

In the present series, the hospital mortality rate was zero and the overall complication rate was $16.7 \%$ with 1 patient had pancreatic fistula, which less than the rates of $33 \%$ reported for aa large series of patients undergoing laparoscopic open D2 total gastrectomy with splenectomy [14]. Like open resection, D2 total gastrectomy with splenectomy for gastric cancer is still debated [14-20]. In few European randomized control trials, splenectomy was strongly associated with increased mortality and morbidity after D2 total gastrectomy [21,22]. Hence, authors in Western countries have recommended that splenectomy be abandoned unless the tumor directly invades the spleen or involves the splenic hilar nodes. In Japan, on the contrary, splenectomy has not been considered an independent determinant of morbidity and mortality associated with D2 total gastrectomy [23]. Lymph node metastasis of gastric cancer is frequently encountered around the suprapancreatic area such as at stations 7, 8, 9, and 11 [24]. Moreover, complete dissection of this area is an important element of curative surgical treatment for gastric cancer [20]. Our technique consists of dissection of the suprapancreatic lymph nodes with initial mobilization of the pancreatic body and downward retraction of the caudal gland of the pancreas by gauze traction to look for the upper border of the pancreas. These steps have helped us to perform meticulous dissection of lymph nodes without injuring the pancreatic parenchyma by using an ultrasonic device or clips if needed. Consequently, the number of lymph nodes removed in our series exceeded 20, indicating that laparoscopic treatment has not compromised adequate lymph node sampling. One of the major concerns in conducting laparoscopic total gastrectomy with D2 lymphadenectomy was the prolonged operation time, which may increase morbidity. However, in our series, the hospital mortality rate was zero and there was acceptable morbidity.

Although laparoscopic surgery has a clear benefit regarding postoperative hospital stay, in our series the hospital stay was relatively long: a median of 16 days overall and maximum 83 days. However, our patients experienced a good postoperative recovery, with a median time to postoperative ambulation of 3 days and a median time to oral feeding of 6 days. This is consistent with the faster recovery noted for laparoscopy-assisted distal gastrectomy for early gastric cancer [4-6]. Therefore, it is possible that postoperative hospital stay will be shorter in the near future.

Themost widespread techniqueforesophagojejunostomy is an anastomosis with a circular stapler prepared for open operation. However, for laparoscopic operation, this technique is complicated with technical difficulty, and a minilaparotomy or an extended auxiliary incision on the abdominal wall for insertion of the circular device may spoil the merit of the totally laparoscopic operation [25]. Our technique for intracorporeal esophagojejunostomy was derived from the application of the functional side-to-side technique to the anastomosis between the antimesenteric side of the distal limb of the jejunum and the posterior side of the esophagus [26]. By means of the described technique, intracorporeal esophagojejunostomy can be performed with the use of a $45-\mathrm{mm}$ linear stapler through a $12-\mathrm{mm}$ low abdominal port without forceful extraction of the surgical specimen. We found that intracorporeal esophagojejunostomy using an endoscopic linear stapler is a simple technique that does not reduce the advantages of laparoscopic gastric resection.

\section{Conclusion:}

The mortality rate of zero and acceptable morbidity of our series shows that laparoscopic total gastrectomy with D1 or D2 lymphadenectomy for gastric cancer is technically feasible and is safe in the hands of experienced surgeons. Although comparative studies with larger sample size, suitable control groups and long term follow up is necessary to confirm this, it yields adequate number of lymph nodes for accurate pathological staging against the anticipation. The findings of our retrospective case series are hoped for contributing to the evidence-based information about the safety, feasibility and outcomes of laparoscopic total gastrectomy for gastric cancer though many future studies are necessary to validate the longterm oncologic outcomes.

\section{Acknowledgements}

We acknowledge the confidence of our patients and support of our Anaesthesia Colleagues and staffs \& support system involved in managing all these cases.

\section{Contributors}

MRS, SS, JN conceptualized and designed the study, acquired, and analyzed data, interpreted the study results, drafted the manuscript, and critically revised the manuscript for important intellectual content. MRS, SS, $\mathrm{JN}$ acquired, and analyzed data, interpreted the study results.

\section{Funding}

No funding was received for this study.

\section{Competing interests}

No benefits in any form have been received or will be received from a commercial party related directly or indirectly to the subject of this article.

\section{Availability of data and materials}

Further information is available from the corresponding author on reasonable request.

Ethics approval

Not applicable 


\section{Provenance and peer review}

Not commissioned; externally peer reviewed.

\section{Open access}

This is an Open Access article distributed in accordance with the Creative Commons Attribution NonCommercial (CC BY-NC 4.0) license, which permits others to distribute, remix, adapt, build upon this work noncommercially, and license their derivative works on different terms, provided

the original work is properly cited and the use is non-commercial. See: http://creativecommons.org/ licenses/by-nc/4.0/

\section{References}

[1] Van Cutsem E, Sagaert X, Topal B, Haustermans K, Prenen H. Gastric cancer. Lancet. 2016; 388 (10060):2654-2664.

[2] Weeks JC, Nelson H, Gelber S, Sargent D, Schroeder G, JAMA. 2002 Jan 16;287(3):321-8.

[3] Strong VE, D'Angelica M, Tang L, Prete F, Gonen M, Coit D, Touijer K, Fong Y, Brennan M. Ann Surg Oncol, 14(12):3392-400, 2007).

[4] Adachi $Y$, Shiraishi N, Shiromizu A, Bandoh T, Aramaki M Kitano S. Laparoscopy-assisted Billroth I gastrectomy compared with conventional open gastrectomy. Arch Surg 2000;135 (7) 806- 810.

[5] Kim MC, Kim KH, Kim HH, Jung GJ. Comparison of laparoscopyassisted by conventional open distal gastrectomy and extraperigastric lymph node dissection in early gastric cancer. J Surg Oncol 2005;91

(1) $90-94$.

[6] Uyama I, Sakurai Y, Komori Y et al. Laparoscopic gastrectomy with preservation of the vagus nerve accompanied by lymph node dissection for early gastric carcinoma. J Am Coll Surg 2005;200 (1) 140145.

[7] Kodera Y, Fujiwara M, Ohashi N, Nakayama G, Koike M, Morita S, Nakao A: Laparoscopic surgery for gastric cancer: a collective review with meta-analysis of randomized trials. J Am Coll Surg 2010; 211:677686.

[8] Son SY, Kim HH. Minimally invasive surgery in gastric cancer. World J Gastroenterol. 2014 Oct;20(39):14132-41.

[9] Japanese Gastric Cancer Association: Japanese gastric cancer treatment guidelines (ver. 3) Gastric Cancer. 2010:113-123. 2011.

[10] Kitano S, Shiraishi N, Uyama I, Sugihara K, Tanigawa N. Japanese Laparoscopic Surgery Study Group, A multicenter study on oncologic outcome of laparoscopic gastrectomy for early cancer in Japan. Ann Surg 2007:245 (1) 68- 72 .

[11] Huscher CG, Mingoli S, Garzini G et al. Laparoscopic versus open subtotal gastrectomy for distal gastric cancer: five-year results of a randomized prospective trial. Ann Surg 2005;241 (2) 232- 237

[12] Usui S, Yoshida T, Ito K, Hiranuma S, Kudo S, Iwai T. Laparoscopyassisted total gastrectomy for early gastric cancer: comparison with conventional open total gastrectomy. Surg Laparosc Endosc Percutan Tech 2005;15 (6) 309- 314 .

[13] Topal B, Leys Etors N, Aerts R, Penninckx F. Determinants of complications and adequacy of surgical resection in laparoscopic versus open total gastrectomy for adenocarcinoma. Surg Endosc 2008;22 (4) 980- 984 .

[14] Shinohara T, Kanaya S, Taniguchi K, Fujita T, Yanaga K, Uyama I. Laparoscopic Total Gastrectomy With D2 Lymph Node Dissection for Gastric Cancer. Arch Surg. 2009;144(12):1138-1142. doi:10.1001/ archsurg.2009.223

[15] Griffith JP, Sue-Ling HM, Martin I et al. Preservation of the spleen improves survival after radical surgery for gastric cancer. Gut 1995;36 (5) 684- 690 .

[16] Otsuji E, Yamaguchi T, Sawai K, Okamoto K, Takahashi T. Total gastrectomy with simultaneous pancreaticosplenectomy or splenectomy in patients with advanced gastric carcinoma. Br J Cancer 1999;79 (11-12) 1789- 1793.

[17] Kasakura Y, Fujii M, Mochizuki F, Kochi M, Kaiga T. Is there a benefit of pancreaticosplenectomy with gastrectomy for advanced gastric cancer? Am J Surg 2000;179 (3) 237- 242.

[18] Yu W, Choi GS, Chung HY. Randomized clinical trial of splenectomy versus splenic preservation in patients with proximal gastric cancer. Br J Surg 2006;93 (5) 559- 563.

[19] Csendes A, Burdiles P, Rojas J, Braghetto I, Diaz J, CMaluenda F. A prospective randomized study comparing $\mathrm{D} 2$ total gastrectomy versus D2 total gastrectomy plus splenectomy in 187 patients with gastric carcinoma. Surgery 2002;131 (4) 401- 407.

[20] Sasako M, McCulloch P, Kinoshita T,Maruyama K. New method to evaluate the therapeutic value of lymph node dissection for gastric cancer. Br J Surg 1995;82 (3) 346- 351

[21] Bonenkamp JJ, Songun I, Hermans J et al. Randomised comparison of mortality after D1 and D2 dissection for gastric cancer in 996 Dutch patients. Lancet1995;345 (8952) 745- 748 .

[22] CuschieriA, Fayers P, Fielding J et al. Surgical Cooperative Group, Postoperative morbidity and mortality after D1 and D2 resections for gastric cancer: preliminary results of the MRC randomized controlled surgical trial. Lancet1996;347 (9007) 995- 999.

[23] Nakajima T. Gastric cancer treatment guidelines in Japan. Gastric Cancer 2002;5 (1) 1- 5

[24] Maruyama K, Gunven PO, kabayashi K, Sasako M, Kinoshita T. Lymph node metastases of gastric cancer: general pattern in 1931 patients. Ann Surg 1989;210(5) 596- 602.

[25] Kanaya S, Gomi T, Momoi H et al. Delta-shaped anastomosis in totally laparoscopic Billroth I gastrectomy: new technique of intraabdominal gastroduodenostomy. J Am Coll Surg 2002;195 (2) 284- 287.

[26] Uyama I, Sugioka A, Fujita J, Komori Y, Matsui H, Hasumi. A Laparoscopic total gastrectomy with distal pancreatosplenectomy and D2 lymphadenectomy for advanced gastric cancer. Gastric Cancer 1999;2 (4) 230- 234. 